\title{
ỨNG DỤNG CỦA MÔ HÌNH LMD-AR VÀ DE-SVM ĐỂ CHẨN ĐOÁN HU' HỎNG Ổ LĂN
}

\author{
AO HÙNG LINH ${ }^{1}$, TRẦN ĐÌNH ANH TUẤN ${ }^{1}$, TRƯONG KHẮC TÙNG ${ }^{1}$, \\ NGUYẼ̃N TRANG THẢO ${ }^{2}$ \\ ${ }^{1}$ Truoòng Đại học Công nghiệp thành phố Hồ Chí Minh, \\ ${ }^{2}$ Truòng Đại học Tôn Đức Thắng; \\ aohunglinh@iuh.edu.vn
}

Tóm tắt. Nghiên cứu này đề xuất một phương pháp mới để chẩn đoán hư hỏng ổ lăn trong đó chúng tôi sử dụng hàm tích $(\mathrm{PF})$ của phương pháp phân rã trung bình cục bộ (LMD) kết hợp với mô hình tự hồi quy (AR) và máy véc tơ hỗ trợ $(\mathrm{SVM})$. Thêm vào đó, các tham số của $\mathrm{SVM}$ được lựa chọn thông qua thuật toán tiến hóa vi phân (DE), ký hiệu DE-SVM. Trước tiên, tín hiệu dao động gia tốc của ổ lăn được phân rã thành những $\mathrm{PF}$ bằng phương pháp $\mathrm{LMD}$. Từ những $\mathrm{PF}$ đó, chúng tôi thiết lập mô hình $\mathrm{AR}$ và trích chọn những hệ số của mô hình thành những véc tơ đầu vào cho bộ phân lớp $\mathrm{SVM}$. Cuối dùng, những bộ phân lớp DE-SVM được dùng để phân loại các mẫu ổ lăn lỗi. Kết quả thực nghiệm cho thấy phương pháp đề xuất có thể phân loại tình trạng hoạt động của ổ lăn với độ chính xác cao và thời gian thấp khi so sánh với các phương pháp khác.

Từ khóa. Tự hồi quy (AR), hàm tích (PF), thuật toán tiến hóa vi phân (DE), phân rã trung bình cục bộ (LMD), máy véc tơ hỗ trợ (SVM).

\section{APPLICATION OF LMD-AR MODEL AND DE-SVM TO DIAGNOSIS ROLLER BEARING FAULT}

\begin{abstract}
This study investigates a new method for roller bearing fault diagnosis based on product functions (PFs) and Autoregressive (AR) model, together with a Support Vector Machine designed using a Differential Evolution (DE) Algorithm, referred to as a DE-SVM. First, the original acceleration vibration signals of roller bearings are decomposed into PFs by using local mean decomposition (LMD) method. Second, the concept of AR model is introduced. Third, AR model is used to extract PFs into feature vectors and served as input vectors for the support vector machine classifier. Finally, the DE-SVM classifiers are proposed to recognize the faulty roller bearing pattern. The experimental analysis results show that the proposed method can classify working condition of roller bearings with higher classification accuracy and lower cost time compared to the other methods.
\end{abstract}

Keywords. Autoregressive, product functions, differential evolution, local mean decomposition, support vector machine.

\section{1. ĐĀT VẤN ĐỀ:}

Ổ lăn là chi tiết tương tác giữa bộ phận cố định và bộ phận quay của máy vì thế ổ lăn được sử dụng rộng rãi trong các ứng dụng công nghiệp như động cơ, hộp số, tuabin, .... Những khuyết tật của ổ lăn sẽ dẫn đến phá hỏng của cả cụm máy hoặc dẫn đến những tai nạn nghiêm trọng. Theo thống kê, $30-40 \%$ hư hỏng của máy móc xuất phát từ ổ lăn bị khuyết tật vì thế chẩn đoán hư hỏng ổ lăn để kịp thời thay thế là rất quan trọng [1].

\section{GIẢI QUYẾT VẤN ĐỀ}

Các dạng khuyết tật chính của ổ lăn bao gồm hư vòng ngoài (OR), hư vòng trong (IR), hư con lăn (B). Nguyền nhân hư hỏng chính có thể do lỗi trong quá trình sản xuât, hao mòn tự nhiên, hao mòn do thay đồi tải đột ngột.

Các phương pháp chẩn đoán khuyết tật ổ lăn bao gồm trực quan, sử dụng dụng cụ đo khoảng hở, kiểm tra bằng siêu âm, kiểm tra bằng tín hiệu âm thanh và chẩn đoán thông qua tín hiệu dao động từ ổ 
lăn. Hai phương pháp đầu tiên cho độ chính xác thấp và độ tin cậy kém. Phương pháp kiểm tra bằng siêu âm đòi kỹ thuật viên giàu kinh nghiệm và cẩn thận khi so sánh sóng siêu âm thu được với sóng mẫu. Thêm vào đó phương pháp này đòi hỏi bề mặt bề mặt chi tiết kiểm tra cần phải lau chùi và cần các khớp nối để truyền năng lượng sóng siêu âm từ vật kiểm tra đến các đầu dò. Phương pháp kiểm tra bằng tín hiệu âm thanh đòi hỏi điều kiện kiểm tra phải cách âm nghiêm ngặt để tránh nhiễu âm thanh, điều này khó ứng dụng trong cơ khí. Phương pháp chẩn đoán thông qua tín hiệu dao động gia tốc được truyền từ các cảm biến gắn trên gối đỡ ổ lăn về bộ thu nhận dữ liệu. Ưu điểm của phương pháp này là dựa trên sự thay đổi khối lượng các chi tiết mà mô đun đàn hồi thay đổi dẫn đến gia tốc quay của các bộ phận ổ lăn cũng thay đổi. Tuy nhiên biên dạng sóng dao động này rất phức tạp, các phương pháp như biến đổi wavelet, biến đổi Fourier chỉ phù hợp với tín hiệu dừng trong khi tín hiệu dao động ổ lăn là tín hiệu không dừng. Trong bài báo này chúng tôi sử dụng hai bước cơ bản là trích xuất đặc tính và nhận dạng khuyết tật. Trước tiên, những tín hiệu dao động gia tốc của những ổ lăn được phân rã thành những hàm tích (product function-PF) bằng phương pháp phân rã trung bình cục bộ (Local mean decomposition-LMD) [2]. Bước thứ hai, mô hình tự hồi quy (Autoregressive-AR) được dùng để trích chọn những hàm tích này thành các véc tơ đặc tính và dùng các véc tơ này làm véc tơ đầu vào cho bộ phân lớp SVM $[2,3]$. Cuối dùng, những bộ phân lớp sử dụng thuật toán tiến hóa vi phân (DE-SVM) được dùng để nhận dạng các mẫu ổ lăn có khuyết tật [4]. Kết quả thực nghiệm cho thấy phương pháp đề xuất có thể phân loại tình trạng hoạt động của ổ lăn với độ chính xác cao và thời gian thấp khi so sánh với các phương pháp khác.

\subsection{Trích xuất đặc tính}

Trích xuất đặc tính là xuất ra các thông số chính của tín hiệu dao động. Trong bài báo này chúng tôi sử dụng phương pháp phân rã trung bình cục bộ kết hợp mô hình tự hồi quy.

\subsubsection{Phương pháp phân rã trung bình cục bộ (LMD)}

Phương pháp LMD được phát triển từ giả thiết là bất kỳ một tín hiệu phức hợp đều bao gồm nhiều $P F$ [2]. Theo phương pháp này, mỗi tín hiệu đều có thể phân tích thành một số $P F$ và một phần dư $r_{n}(t)$.

$$
x(t)=\sum_{p=1}^{n} P F_{p}(t)+r_{n}(t)
$$

với $p$ là số lượng $P F, r_{n}$ là một hàm đơn điệu.

\subsubsection{Mô hình tự hồi quy (AR)}

Mô hình AR là một mô hình cơ bản trong phân tích chuỗi thời gian. Tương tự như hồi quy đa biến, $\mathrm{AR}$ là một mô hình hồi quy, tuy nhiên biến phụ thuộc và biến độc lập trong $\mathrm{AR}$ lần lượt là giá trị tại thời điểm $t$ và giá trị $p$ trước của chuỗi thời gian $(\mathrm{lag}=p)$. Một mô hình $\mathrm{AR}$ thường có dạng như sau:

$$
X_{t}=\sum_{i=1}^{p} a_{i} X_{t-i}+\varepsilon_{t} ; \quad a_{p} \neq 0
$$

với $p$ là bậc của tự hồi quy, $X_{\mathrm{t}-\mathrm{i}}$ là giá trị của chuỗi thời gian tại lag=i, $a_{\mathrm{i}}$ là hệ số của $X_{\mathrm{t}-\mathrm{i}}, \varepsilon_{t}$ là lỗi ngẫu nhiên. Mô hình $A R$ bao gồm ba bước là nhận dạng mô hình, ước lượng hệ số và kiểm tra chẩn đoán.

\subsection{Nhận dạng khuyết tật}

Các thông số chính của tín hiệu dao động thu được từ bước trích xuất đặc tính được đưa vào bộ nhận dạng máy véc tơ hỗ trợ (SVM). Tuy nhiên máy véc tơ hỗ trợ có khuyết điểm là việc lựa chọn hai tham số $\mathrm{C}$ và $\sigma$ đòi hỏi kinh nghiệm của người dùng. Trong bài báo này chúng tôi sử dụng thuật toán tiến hóa vi phân để tối ưu hai thông số trên.

\subsubsection{Máy véc tơ hỗ trọ̣}

SVM là kỹ thuật học máy mạnh dựa trên lý thuyết học thống kê. Ý tưởng cơ bản của SVM là ánh xạ những mẫu huấn luyện từ không gian đầu vào vào một không gian đặc tính có số chiều cao hơn bằng cách sử dụng một hàm ánh xạ $\phi$ [3]. Giả thiết rằng cho trước một tập mẫu huấn luyện $\left.G=\left\{x_{i}, y_{i}\right\}, i=1,2, \ldots, l\right\}$, với mỗi mẫu thuộc một lớp $y \in\{+1,-1\}$ và dữ liệu huấn luyện phi tuyến không thể phân chia trong không gian đặc tính, hàm mục tiêu có thể được biểu diễn như sau [5]: 


$$
\left\{\begin{array}{cc}
\operatorname{minimize} & \phi(\omega)=\frac{1}{2}\langle\omega . \omega\rangle+C \sum_{i=1}^{l} \xi_{i} \\
\text { s.t } \quad y_{i}(\langle\omega . \phi(\omega)\rangle+b) \geq 1-\xi_{i}, & \xi_{i} \geq 0, \quad i=\{1,2, \ldots, l\}
\end{array}\right.
$$

với $\omega$ là véc tơ pháp tuyến của siêu mặt; $C$ là tham số; $b$ là độ lệch và là một vô hướng; $\xi_{i}$ là những biến bù không âm; $\phi(x)$ là hàm ánh xạ.

Đưa vào những nhân tử Lagrange $\alpha_{i} \geq 0$, bài toán tối ưu có thể được viết lại như sau:

$$
\begin{array}{ll}
\max & L(\omega, b, \alpha)=\sum_{i=1}^{l} \alpha_{i}-\frac{1}{2} \sum_{i, j=1}^{l} \alpha_{i} \alpha_{i} y_{i} y_{j} K\left(x_{i}, x_{j}\right) \\
\text { s.t } \quad & \sum_{i=1}^{l} \alpha_{i} y_{i}=0, \quad 0 \leq \alpha_{i} \leq C, i=1,2, \ldots, l
\end{array}
$$

với $K\left(x_{i}, x_{j}\right)$ là hàm kernel.

Hàm quyết định siêu phẳng tối ưu (hyperplan) có thể thu được theo [3]

$$
f(x)=\operatorname{sgn}\left[\sum_{i=1}^{l} \alpha_{i} y_{i} K\left(x, x_{i}\right)+b\right\rfloor
$$

Hàm kernel thường dùng trong SVM là hàm RBF (radial basis function), được biểu diễn trong công thức sau $[5,6]$

$$
K\left(x, x_{i}\right)=\exp \left(-\left\|x-x_{i}\right\|^{2} / 2 \sigma^{2}\right)
$$

với $\sigma$ là thông số kernel.

Thông số $C$ trong công thức (3) và $\sigma$ trong công thức (6) được chọn lựa bằng thuật toán $\mathrm{DE}$.

\subsubsection{Thuật toán tiến hóa vi phân (DE)}

Thuật toán DE là một thuật toán tìm kiếm trực tiếp song song dựa trên những di truyền cơ bản bao gồm đột biến, lai ghép và lựa chọn để tạo ra những cá thể thử [4]. Thuật toán DE bao gồm năm bước sau: (i) Định nghĩa bài toán và những thông số của thuật toán; (ii) Khởi tạo; Đột biến; (iv) Lai ghép; $(v)$ Lựa chọn. Tiến trình của thuật toán DE được biểu diễn như trong Hình 1.

\section{Định nghĩa bài toán và các thông số của thuật toán}

Cực tiểu hàm $f(x)$

sao cho $\mathbf{x}_{i} \in D_{i}=\left[l_{i}, u_{i}\right], i=1,2, \ldots, M$

Trong công thức trên, $f(x)$ là một hàm mục tiêu (fitness function); $\mathbf{x}=\left(x_{1}, x_{2}, \ldots, x_{N}\right)$ là véc tơ các biến độc lập; $N$ là số lượng biến độc lập; $D_{i}, l_{i}$ và $u_{i}$ lần lượt là vùng chứa giá trị khả thi, biên dưới và biên trên của biến độc lập thứ $i$;

\subsubsection{Tối ưu hóa thông số SVM bằng thuật toán DE}

Những thông số SVM tối ưu sẽ làm tăng độ chính xác phân loại. Những thông số hàm kernel Gaussian bao gồm một nhân tố phạt $C$ và thông số kernel $\sigma$. Việc lựa chọn những thông số $\mathrm{SVM}$ không phải là công việc dễ dàng. Thông thường, $C$ và $\sigma$ được lựa chọn dựa trên kinh nghiệm vì thế dẫn đến những kết quả phân loại không tối ưu. Trong bài báo này thuật toán $\mathrm{DE}$ được dùng để tối ưu $C$ và $\sigma$. Hàm mục tiêu là lỗi kiểm tra của SVM. Lỗi kiểm tra của SVM được biểu diễn như sau:

$$
G(x)=L K T_{S V M}(x)
$$

trong đó $G(x)$ hàm mục tiêu, $L K T_{S V M}$ là lỗi kiểm tra và $x=\{C, \sigma\}$,

Trong công thức (8) lỗi kiểm tra của SVM được định nghĩa:

$L K T_{S V M}=$ Số lượng mẫu sai trong nhũng mẫu kiểm tra/Tổng số của mẫu trong tập kiểm tra 
Mô hình DE-SVM bao gồm bốn bước, được biểu diễn như Hình 2: $(i)$ Khởi tạo những giá trị $\mathrm{C}$ và $\sigma$, (ii) Huấn luyện những tập dữ liệu mẫu với những giá trị $C$ và $\sigma$ được tạo ra. (iii) Tính toán hàm mục tiêu; (iv) Lập lại vòng lập cho đến khi đạt được điều kiện dừng.

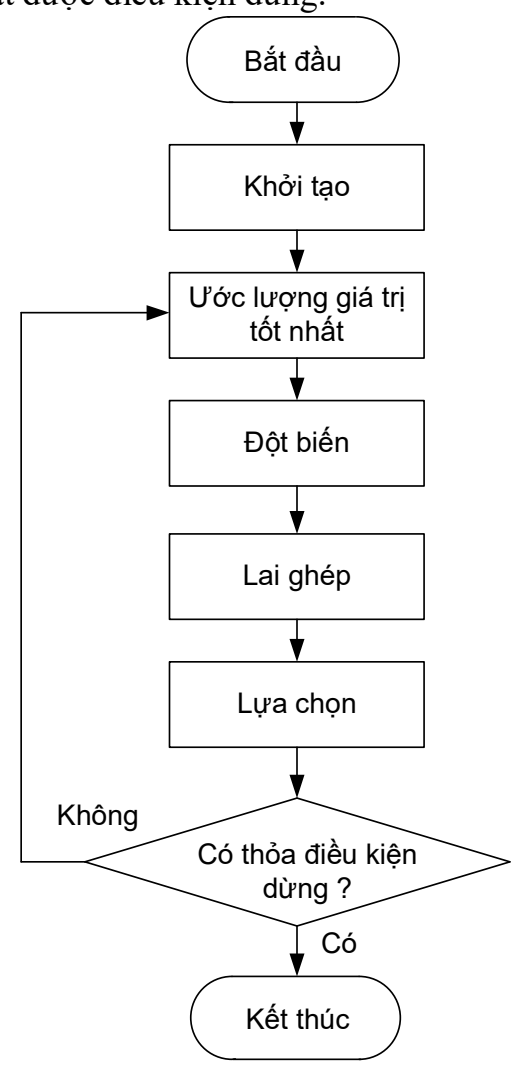

Hình 1. Sơ đồ khối của thuật toán DE.

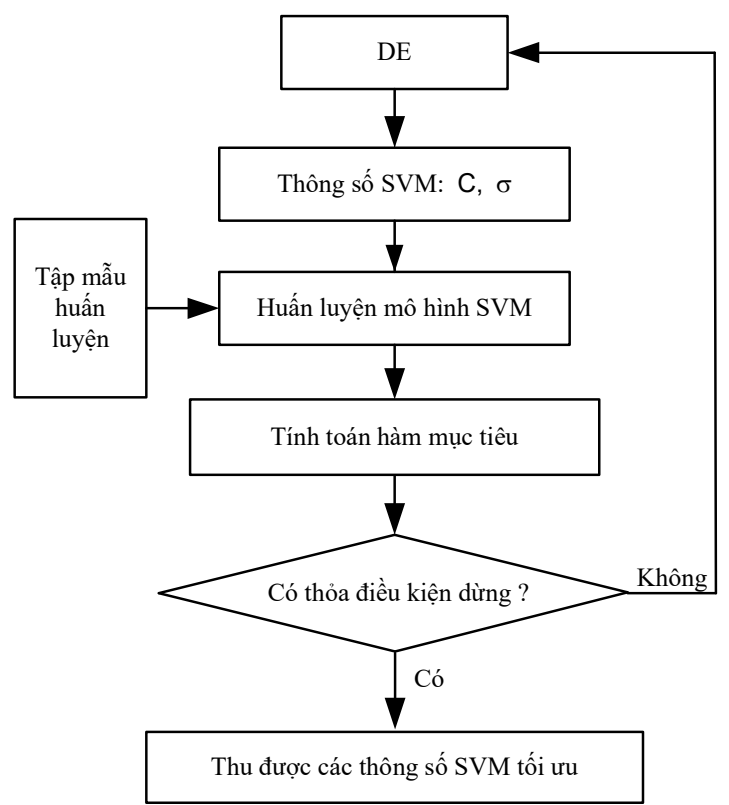

Hinh 2. Sơ đồ thuật toán DE-SVM. 


\section{THỰC NGHIỆM}

Sơ đồ giải thuật của phương pháp chẩn đoán trong bài báo này được minh họa trong Hình 3. Các bước tiến hành thực nghiệm như sau:

(1) Dùng bộ đo gia tốc dao động, lấy mẫu $N$ lần tại một tần số lấy mẫu cố định dưới những điều kiện làm việc như điều kiện bình thường, hư vòng ngoài, hư vòng trong, tuần tự. Tất cả những mẫu tín hiệu dao động được chia thành hai nhóm gồm nhóm mẫu huấn luyện và kiểm tra.

(2) Mỗi tín hiệu mẫu được phân rã bởi phương pháp LMD và thu được chuỗi các $P F$.

(3) Để giới hạn hiệu ứng của biên độ tín hiệu với sự khác nhau của phần dư $\sigma_{i}^{2}$, chúng tôi chuẩn hóa mỗi thành phần $P F$ để đạt được một thành phần mới.

$$
\hat{P F}=\frac{P F_{i}(t)}{\sqrt{\int_{-\infty}^{\infty} P F_{i}^{2}(t)}}
$$

(4) Ước lượng mô hình $\mathrm{AR}$ bậc $m$ cho thành phần đã chuẩn hóa, các hệ số tự hồi quy $\varphi_{i k}(k=1,2, \ldots, m)$ và phương sai của phần dư $\sigma_{i}^{2}$, với $\varphi_{i k}$ là hệ số tự hồi quy thứ $k$ của thành phần $\mathrm{PF}$ thứ $i$. Vì thế, véc tơ đặc tính được dùng như là véc tơ đầu vào của SVM được biểu diễn như sau: $A_{i}=\left[\varphi_{i 1}, \varphi_{i 2}, \ldots, \varphi_{i m}, \sigma_{i}^{2}\right]$.

(5) Thiết kế bộ phân lớp DE-SVM và nhận dạng hư hỏng của ổ lăn.

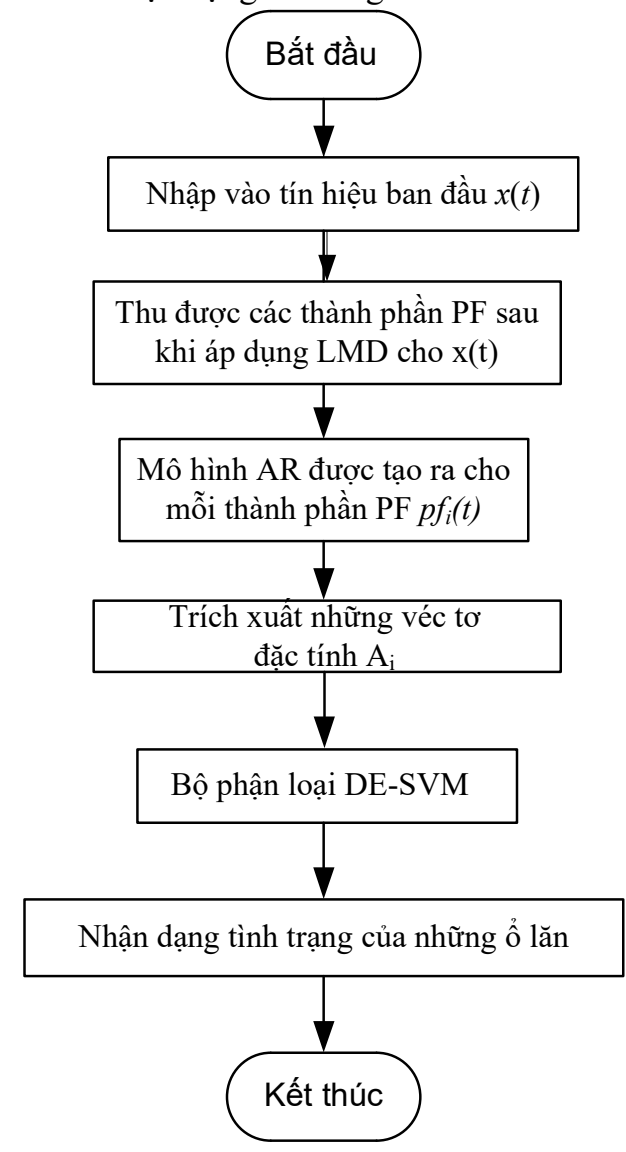

Hình 3. Sơ đồ thuật toán LMD-AR và DE-SVM.

\subsection{Thu thập dữ liệu}

Dữ liệu được thu thập dựa trên băng thử nhỏ thể hiện trong Hình 4 , băng thử này thường dùng để kiểm tra độ cân bằng, độ thẳng hàng và những khuyết tật khác của ổ lăn. Băng thử này bao gồm một động cơ, một khớp nối, một rô to và một trục với hai ổ lăn. Những ổ lăn là loại ký hiệu 6311 . Tần số quay của 
trục là $25 \mathrm{~Hz}$ và mô men quán tính độc cực của rô to là $0.03 \mathrm{~kg} \cdot \mathrm{m}^{2}$. Bằng thực nghiệm ba tần số cộng hưởng đầu tiên được xác định tuần tự là $420 \mathrm{~Hz}, 732 \mathrm{~Hz}$ và $1016 \mathrm{~Hz}$. Vì thế tần số lấy mẫu được lấy ở $4096 \mathrm{~Hz}$.

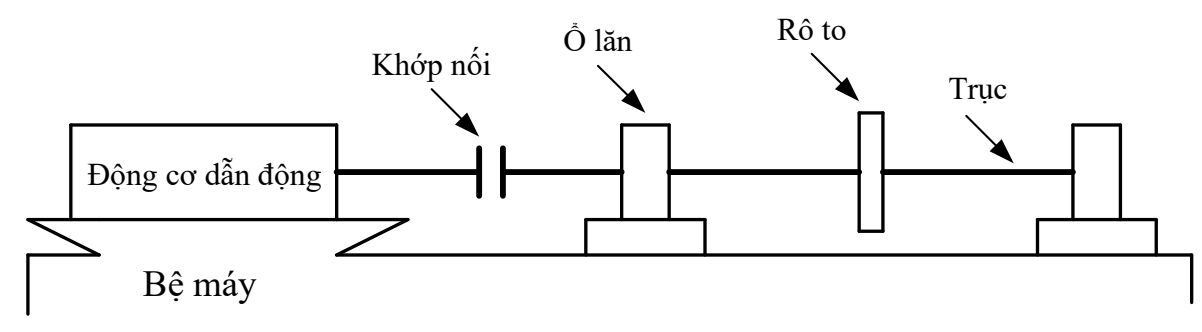

Hình 4. Băng thử

Tín hiệu dao động được thu thập từ cảm biến gia tốc được gắn trên gối đỡ ổ lăn tại tần số cố định của trục. Vì ổ lăn thường được dùng ở tốc độ cố định, quá trình khởi động và dừng có thể được bỏ qua. Ổ lăn hỏng được tạo ra bằng cách dùng phương pháp laser cắt một rãnh rộng $0.15 \mathrm{~mm}$ và sâu $0.13 \mathrm{~mm}$. Ba tình trạng của ổ lăn (bình thường (NOR), hư vòng trong (IR) và hư vòng ngoài $(\mathrm{OR})$ ) được kiểm tra và thu thập 45 tín hiệu dao động của ổ lăn ở mỗi tình trạng. Chọn ngẫu nhiên 30 mẫu tín hiệu để huấn luyện và phần còn lại dùng để kiểm tra.

\section{2. Úng dụng}

Trước tiên, những tín hiệu dao động được phân rã thành một số $P F$ bằng phương pháp LMD. Lưu ý rằng phân tích ở các nghiên cứu $[7,8]$ trước cho thấy ba thành phần $P F$ đầu tiên của tần số cao chứa đựng thông tin lỗi quan trọng của ổ lăn. Vì thế, ma trận véc tơ đặc tính khởi tạo được ước lượng với bốn thành phần $\mathrm{PF}$ đầu tiên của tần số cao hơn. Thứ hai, áp dụng mô hình $\mathrm{AR}$ cho ma trận véc tơ khởi tạo từ đó có thể thu được những giá trị riêng tương ứng - những giá trị liên quan đến véc tơ đầu vào cho bộ phân lớp DE-SVM. Thứ ba, với những véc tơ đầu vào từ những mẫu huấn luyện, những bộ phân loại DE-SVM được thiết kế để đạt được việc phân loại đa lớp như Hình 5. Cuối cùng, những mẫu chưa biết được phân lớp với DE-SVM để nhận dạng những tình trạng ổ lăn. Với SVM1, nhận dạng tình trạng hoạt động với lỗi hư vòng ngoài là $y=+1$ và những tình trạng khác là $y=-1$; vì thế lỗi hư vòng ngoài có trong có thể được tách ra khỏi những tình trạng khác. Kế tiếp SVM2 sẽ tách tình trạng hư vòng trong và phần còn lại là ổ lăn bình thường.

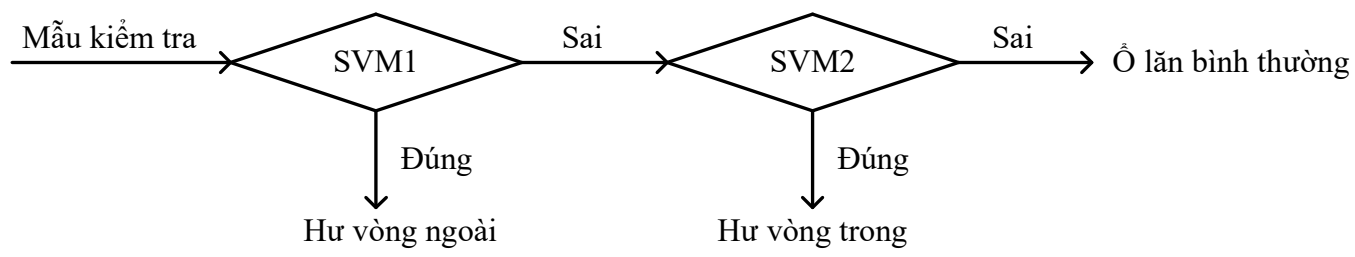

Hinh 5. Sơ đồ thuật toán chẩn đoán hư hỏng ổ lăn bằng nhiều bộ phân lớp SVM.

\section{KẾT QUẢ VÀ THẢO LUẬN}

Bảng 1 trình bày chi tiết kết quả phân loại tình trạng ổ lăn bằng phương pháp LMD-AR-DE-SVM. Tình trạng của ổ lăn được chia làm 3 loại, lần lượt là hư vòng ngoài, hư vòng trong và bình thường. Kết quả cho thấy tín hiệu dao động của ổ lăn sau khi được phân rã thành từng PF kết hợp với trích chọn các thông số bởi mô hình $\mathrm{AR}$ đã tạo giá trị đầu vào tốt cho bộ phân lớp SVM. Thêm vào đó, bộ phân lớp SVM với các thông số đã được tối ưu bởi thuật toán $\mathrm{DE}$ đã phân loại các mẫu kiểm tra thành từng nhóm riêng biệt. Để thấy rõ sự khác biệt, chúng tôi cũng đồng thời thiết kế bộ phân lớp GA-SVM và PSOSVM. Bảng 2 thể hiện kết quả phân lớp trên tập kiểm tra của các phương pháp được sử dụng. Theo đó, 
kết quả ở Bảng 2 cho thấy phương pháp đề xuất cho kết quả phân loại cao hơn cùng với thời gian ngắn hơn so với các phương pháp như LMD-AR-GA-SVM và LMD-AR- PSO-SVM.

\section{KẾT LUẬN}

Bảng 1. Kết quả phân loại hư hỏng ổ lăn bằng phương pháp LMD-AR và DE-SVM.

\begin{tabular}{|c|c|c|c|c|c|c|c|c|}
\hline $\begin{array}{l}\text { Tình trạng } \\
\text { của ổ lăn }\end{array}$ & $\mathrm{PF}$ & $\varphi_{i 1}$ & $\varphi_{i 2}$ & $\varphi_{i 3}$ & $\sigma_{i}^{2}$ & $\begin{array}{l}\text { LMD-AR- } \\
\text { DE-SVM1 }\end{array}$ & $\begin{array}{c}\text { LMD- } \\
\text { AR-DE- } \\
\text { SVM2 }\end{array}$ & $\begin{array}{l}\text { Kết quả } \\
\text { phân lớp }\end{array}$ \\
\hline \multirow{3}{*}{$\begin{array}{c}\text { Hư vòng } \\
\text { ngoài }\end{array}$} & $p f_{1}$ & -0.2616 & -0.1835 & -0.1709 & 0.9107 & +1 & & \multirow{3}{*}{ OR } \\
\hline & $p f_{2}$ & 2.1597 & -2.4306 & 1.8468 & 0.074 & +1 & & \\
\hline & $p f_{3}$ & 3.9575 & -8.1742 & 11.799 & 0.0036 & +1 & & \\
\hline \multirow{3}{*}{$\begin{array}{l}\text { Hư vòng } \\
\text { ngoài }\end{array}$} & $p f_{1}$ & -0.2791 & -0.4028 & 0.0722 & 0.4766 & +1 & & \multirow{3}{*}{ OR } \\
\hline & $p f_{2}$ & 2.0677 & -2.475 & 1.9365 & 0.0544 & +1 & & \\
\hline & $p f_{3}$ & 4.6111 & -10.5019 & 16.0504 & 0.0002 & +1 & & \\
\hline \multirow{3}{*}{$\begin{array}{l}\text { Hư vòng } \\
\text { ngoài }\end{array}$} & $p f_{1}$ & -0.3397 & -0.4283 & 0.0798 & 0.5729 & +1 & & \multirow{3}{*}{ OR } \\
\hline & $p f_{2}$ & 1.7736 & -1.3711 & 0.4208 & 0.1107 & +1 & & \\
\hline & $p f_{3}$ & 4.2862 & -8.7313 & 11.1701 & 0.0004 & +1 & & \\
\hline \multirow{3}{*}{$\begin{array}{c}\text { Hư vòng } \\
\text { ngoài }\end{array}$} & $p f_{1}$ & -0.2418 & -0.6641 & 0.1863 & 0.4248 & +1 & & \multirow{3}{*}{ OR } \\
\hline & $p f_{2}$ & 1.9404 & -1.5529 & 0.4526 & 0.0646 & +1 & & \\
\hline & $p f_{3}$ & 3.7698 & -6.7083 & 7.2314 & 0.0023 & +1 & & \\
\hline \multirow{3}{*}{$\begin{array}{l}\text { Hư vòng } \\
\text { ngoài }\end{array}$} & $p f_{1}$ & -0.1498 & -0.6022 & 0.3022 & 0.43 & +1 & & \multirow{3}{*}{ OR } \\
\hline & $p f_{2}$ & 1.8595 & -1.8237 & 1.1529 & 0.0655 & +1 & & \\
\hline & $p f_{3}$ & 3.9888 & -7.3312 & 8.6686 & 0.0001 & +1 & & \\
\hline \multirow{3}{*}{$\begin{array}{l}\text { Hư vòng } \\
\text { trong }\end{array}$} & $p f_{l}$ & -0.6805 & -0.3328 & -0.0141 & 0.6625 & -1 & +1 & \multirow{3}{*}{ IR } \\
\hline & $p f_{2}$ & 1.6667 & -1.2323 & 0.2015 & 0.052 & -1 & +1 & \\
\hline & $p f_{3}$ & 3.9118 & -6.5739 & 5.9576 & 0.0004 & -1 & +1 & \\
\hline \multirow{3}{*}{$\begin{array}{l}\text { Hư vòng } \\
\text { trong }\end{array}$} & $p f_{1}$ & -0.527 & -0.2527 & 0.0388 & 0.7605 & -1 & +1 & \multirow{3}{*}{ IR } \\
\hline & $p f_{2}$ & 1.9899 & -1.6894 & 0.526 & 0.0533 & -1 & +1 & \\
\hline & $p f_{3}$ & 3.1451 & -4.9996 & 5.1397 & 0.0117 & -1 & +1 & \\
\hline \multirow{3}{*}{$\begin{array}{l}\text { Hư vòng } \\
\text { trong }\end{array}$} & $p f_{1}$ & -0.8091 & -0.3802 & -0.1814 & 0.5741 & -1 & +1 & \multirow{3}{*}{ IR } \\
\hline & $p f_{2}$ & 1.5947 & -1.2734 & 0.4179 & 0.1712 & -1 & +1 & \\
\hline & $p f_{3}$ & 4.2063 & -8.7308 & 11.9513 & 0.0011 & -1 & +1 & \\
\hline \multirow{3}{*}{$\begin{array}{l}\text { Hư vòng } \\
\text { trong }\end{array}$} & $p f_{1}$ & -0.9768 & -0.4391 & -0.12 & 0.4412 & -1 & +1 & \multirow{3}{*}{ IR } \\
\hline & $p f_{2}$ & 1.7375 & -1.335 & 0.4221 & 0.1219 & -1 & +1 & \\
\hline & $p f_{3}$ & 4.3322 & -9.7677 & 15.1724 & 0.0016 & -1 & +1 & \\
\hline \multirow{3}{*}{$\begin{array}{l}\text { Hư vòng } \\
\text { trong }\end{array}$} & $p f_{1}$ & -0.5338 & -0.5779 & 0.0264 & 0.5737 & -1 & +1 & \multirow{3}{*}{ IR } \\
\hline & $p f_{2}$ & 1.7493 & -1.4344 & 0.434 & 0.1097 & -1 & +1 & \\
\hline & $p f_{3}$ & 3.9391 & -7.7467 & 9.0307 & 0.0014 & -1 & +1 & \\
\hline \multirow{3}{*}{ Bình thường } & $p f_{1}$ & -0.3596 & -0.4551 & 0.0424 & 0.7267 & -1 & -1 & \multirow{3}{*}{ NOR } \\
\hline & $p f_{2}$ & 1.5754 & -1.5248 & 0.7455 & 0.1546 & -1 & -1 & \\
\hline & $p f_{3}$ & 3.7091 & -6.0068 & 5.3649 & 0.0006 & -1 & -1 & \\
\hline & $p f_{1}$ & -0.4179 & -0.4621 & 0.0081 & 0.7199 & -1 & -1 & \\
\hline Bình thường & $p f_{2}$ & 1.7602 & -1.4444 & 0.494 & 0.1217 & -1 & -1 & NOR \\
\hline & $p f_{3}$ & 4.4182 & -9.1139 & 11.5665 & 0.0003 & -1 & -1 & \\
\hline & $p f_{1}$ & -0.2719 & -0.4356 & -0.0174 & 0.7877 & -1 & -1 & \\
\hline Bình thường & $p f_{2}$ & 1.638 & -1.2583 & 0.3806 & 0.1516 & -1 & -1 & NOR \\
\hline & $p f_{3}$ & 4.0135 & -7.8284 & 9.5782 & 0.0015 & -1 & -1 & \\
\hline & $p f_{1}$ & -0.279 & -0.4864 & 0.0482 & 0.7181 & -1 & -1 & \\
\hline Bình thường & $p f_{2}$ & 1.512 & -1.1888 & 0.3518 & 0.1888 & -1 & -1 & NOR \\
\hline & $p f_{3}$ & 2.4601 & -2.6773 & 1.6089 & 0.0205 & -1 & -1 & \\
\hline Bình thường & $p f_{l}$ & -0.2669 & -0.4954 & 0.0304 & 0.7199 & -1 & -1 & $\mathrm{NOR}$ \\
\hline Binn tnương & $p f_{2}$ & 1.6706 & -1.3242 & 0.3787 & 0.1283 & -1 & -1 & NUK \\
\hline
\end{tabular}


Bài báo đã đề xuất một mô hình chẩn đoán hư hỏng ổ lăn dựa trên máy vec tơ hỗ trợ SVM kết hợp với phương pháp trích chọn đặc trưng $\mathrm{AR}$. Bên cạnh đó, các thông số của $\mathrm{SVM}$ cũng được tối ưu bằng thuật toán $\mathrm{DE}$, điều này làm rút ngắn thời gian phân lớp và tăng cường độ chính xác của bộ phân lớp SVM. Các tín hiệu dao động ổ lăn được phân tích thành những $P F$ bằng phương pháp LMD tạo thuận lợi cho việc trích chọn các thông số đặc trưng bằng mô hình $\mathrm{AR}$ ở bước tiếp theo. Các kết quả thực nghiệm của chúng tôi cho thấy việc áp dụng phương pháp phân lớp DE-SVM kết hợp với việc trích chọn các thông số đặc trưng bằng mô hình $\mathrm{AR}$ vào bài toán chẩn đoán khuyết tật ổ lăn đạt độ chính xác cao và thời gian ngắn hơn khi so sánh với các phương pháp LMD-AR-PSO-SVM hoặc LMD-AR-GA-SVM.

Bảng 2. Kết quả phân lớp hư hỏng ổ lăn của phương pháp LMD-AR-DE-SVM so sánh với phương pháp LMD-ARGA-SVM và LMD-AR-PSO-SVM.

\begin{tabular}{|rcccccc|}
\hline Phương pháp & $\begin{array}{c}\text { Mẫu huấn } \\
\text { luyện }\end{array}$ & $\begin{array}{c}\text { Mẫu kiểm } \\
\text { tra }\end{array}$ & $\begin{array}{c}\text { Thông số } C \\
\text { tối ưu }\end{array}$ & $\begin{array}{c}\text { Thông số } \\
\sigma \text { tối ưu }\end{array}$ & Thời gian (s) & $\begin{array}{c}\text { Mức độ lỗi } \\
\text { trung bình (\%) }\end{array}$ \\
\hline $\begin{array}{r}\text { LMD-AR-DE- } \\
\text { SVM1 }\end{array}$ & 45 & 15 & 1994.80 & 2.89 & $\mathbf{2 . 3 7}$ & $\mathbf{0}$ \\
$\begin{array}{r}\text { LMD-AR-PSO- } \\
\text { SVM1 }\end{array}$ & 45 & 15 & 399.97 & 7.63 & 3.15 & 1.46 \\
$\begin{array}{r}\text { LMD-AR-GA- } \\
\text { SVM1 }\end{array}$ & 45 & 15 & 29147.78 & 0.11 & 3.21 & 2.78 \\
\hline $\begin{array}{r}\text { LMD-AR-DE- } \\
\text { SVM2 }\end{array}$ & 20 & 10 & 113.84 & 7.88 & $\mathbf{4 . 1 3}$ & $\mathbf{0}$ \\
$\begin{array}{r}\text { LMD-AR-PSO- } \\
\text { SVM2 }\end{array}$ & 20 & 10 & 320.15 & 20.40 & 4.42 & 1.21 \\
LMD-AR-GA- \\
SVM2
\end{tabular}

\section{TÀI LIỆ THAM KHẢO}

[1] Randall R.B., Antoni J., "Rolling element bearing diagnostics-A tutorial", Mechanical Systems and Signal Processing, 25 (2011) 485-520.

[2] Smith J.S., "The local mean decomposition and its application to EEG perception data", Journal of The Royal Society Interface, 2 (2005) 443-454.

[3] Zhang X., Chen X., He Z., "An ACO-based algorithm for parameter optimization of support vector machines", Expert Systems with Applications, 37 (2010) 6618-6628.

[4] Storn R., Price K., "Differential Evolution - A simple and efficient adaptive scheme for global optimization over continuous spaces", Journal of Global Optimization, (1995).

[5] Vapnik V.N., The Nature of Statistical Learning Theory; Springer: New York, 1995.

[6] Aizerman A., Braverman E.M., Rozoner L.I., "Theoretical foundations of the potential function method in pattern recognition learning", Automation and Remote Control, 25 (1964) 821-837.

[7] Ao H., Cheng J., Yang Y., Truong T.K., "The support vector machine parameter optimization method based on artificial chemical reaction optimization algorithm and its application to roller bearing fault diagnosis", Journal of Vibration and Control, 21 (2013) 2434-2445.

[8] HungLinh Ao T.N.T., V. Ho Huu, Linh Anh-Le and TrangThao Nguyen, "Backtracking Search Optimization Algorithm and its Application to Roller Bearing Fault Diagnosis", International Journal of Acoustics and Vibration, 4 (2016) 445-452.

Ngày nhận bài: 13/06/2017 Ngày chấp nhận đăng: 29/12/2017 